\title{
Rhabdomyosarcoma of the Infratemporal Fossa: A Case Report
}

Toufga $Z^{1 *}$, Fikri $M^{1,2}$ and J iddane $M^{1,2}$

${ }^{1}$ Department of Neuroradiology, Military Training

Hospital Mohamed V, Morocco

${ }^{2}$ Faculty of Medicine and Pharmacy, University

Mohammed V, Morocco

*Corresponding author: Zakaria Toufga, Department of radiology, Military Training Hospital Mohamed V, avenue of FAR, hay Riyad, 10100, Rabat, Morocco

Received: February 07, 2018; Accepted: March 12, 2018; Published: March 22, 2018

\section{Introduction}

Rhabdomyosarcoma is a rare malignant tumor; in adults, soft tissue sarcomas account for less than $1 \%$ of all adult malignancies, and rhabdomyosarcoma accounts for 3\% of all soft tissue sarcomas [1]. In our case we report a localization in the infratemporal fossa that is rarely described in the literature.

\section{Case Description}

A 40-year-old woman with no significant history who has had intense and insomniating right jaw pains for 4 months, associated with right facial tingling, and discomfort with chewing; the evolution was marked by the appearance of a right parotid swelling, and a limitation of the opening of the mouth without notion of fever.

The clinical examination found a slightly painful right parotid mass, which has a hard consistency, difficult to limit and which raises the lobule of the ear without signs of facial paralysis

The biological tests were without particularities.

Ultrasound revealed nodular, hypoechogenic and heterogeneous intraparotid formation with irregular contours, and moderate Doppler color vascularity.

Magnetic Resonance Imaging (MRI) revealed a tumor mass of the right infratemporal fossa, developed in the lateral pterygoid muscle, which has a tissue signal on T2-weighted sequences (Figure 1), enhanced after injection of gadolinium (Figure 2), repressing the deep lobe of the parotid and the internal carotid, and responsible for lysis of the internal cortex of the ascending branch of the adjacent mandible.

The patient was operated on and the result of the histopathological examination was in favor of a pleomorphic rhabdomyosarcoma; evolution after chemotherapy was more or less favorable.

\section{Discussion}

Rhabdomyosarcoma is a malignant soft tissue tumor that develops from striated muscle cells, localization in the head and neck is relatively common in children while it is rare in adults [2]; the orbit is the most frequent site of origin in this region followed by the nasosinusal region; the infra-temporal fossa is an extremely rare presentation site [3].

There are three histological subtypes: embryonic, alveolar and pleomorphic; In children, about $60 \%$ are embryonic tumors and $20 \%$ are alveolar tumors; the pleomorphic form occurs mainly in adults [3].

The clinical signs depend on the location and volume of the tumor; it can be asymptomatic as it can be manifested by rhinorrhea, recurrent otitis media, exophthalmia, headache, pain or other functional disorders [4].

Computed Tomography (CT) usually shows a mass with irregular contours, relatively homogeneous, generally enhanced to the same degree as the muscle after injection of contrast medium, without calcification or haemorrhagic lesion, associated with osteolysis of adjacent bone structures [5].

Although computed tomography and magnetic resonance imaging are complementary, MRI remains the test of choice because of its better soft tissue contrast, excellent spatial resolution, multiplanar imaging, and absence of radiation ionizing; it makes it possible to better evaluate the reports of the tumor with respect to the base of the skull and the vascular axes, to confirm the presence or not of a meningeal attack, and to avoid some useless artefacts. It shows a mass with irregular contours, invasive in most cases, which has an intense signal in T2-weighted sequence, moderate signal in T1-weighted sequence, enhanced after gadolinium injection [6]. The differentiation of rhabdomyosarcoma subtype characteristics remains difficult in terms of imaging.

The differential diagnosis with other malignant tumors of the head and neck remains difficult, however, the imaging results show for example that the lymphoma is a little different from the rhabdomyosarcoma in its multifocal involvement and less often associated with the invasion and the destruction of adjacent bone structures; Liposarcomas generally have a component of fat density/ intensity; chondroma, chondrosarcoma and osteosarcoma usually have areas of calcification [5].

The treatment depends on the site and size of the tumor, the 


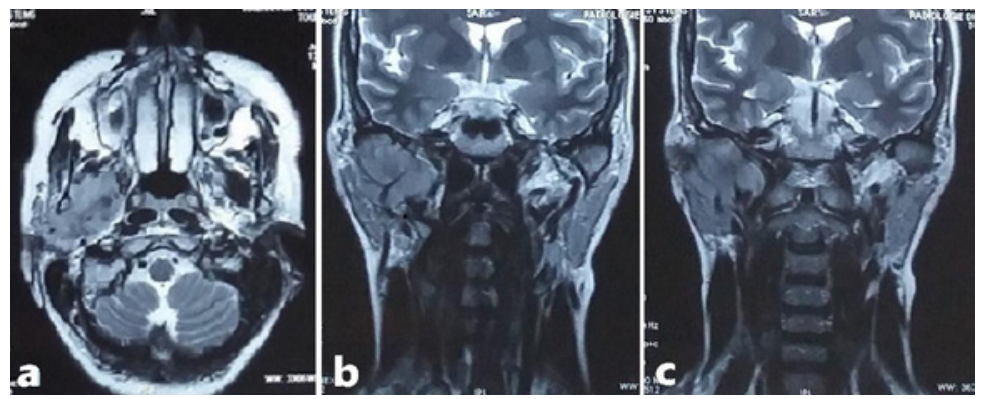

Figure 1: a: axial section in weighted sequences T2; b;c: Coronal section in weighted sequences T2: showing a tumor mass of the right infratemporal fossa, which has a tissue signal, developed at the expense of the lateral pterygoid muscle, measuring about $4 \mathrm{~cm}$ in transverse diameter and $3.5 \mathrm{~cm}$ in height. It comes into contact with the parapharyngeal fat and pushes the internal carotid and the deep lobe of the parotid with a lysis of the internal cortex of the ascending branch of the mandible.

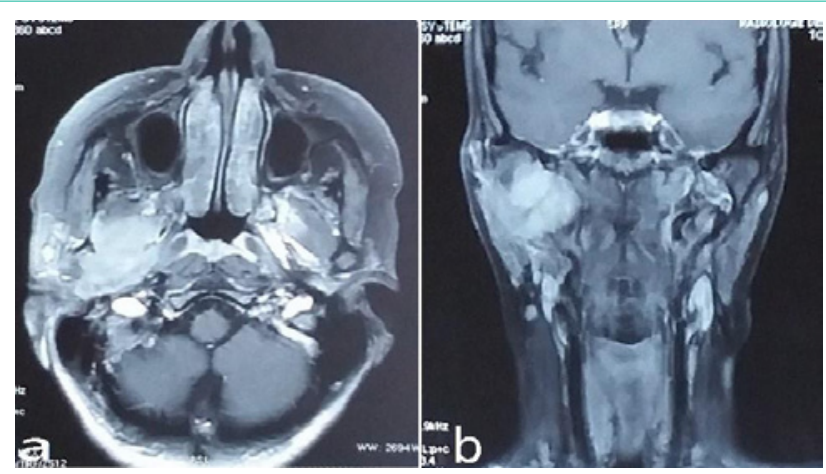

Figure 2: a: axial section and b: coronal section in weighted sequences T1 with gadolinium injection showing the intense enhancement of the tumor after injection

histological subtype, the age of the patient and the extent of the disease, some recommend first surgery if there is no consequence functional and / or aesthetic, followed by chemotherapy. Patients with initially unresectable tumors may benefit from surgery to remove residual tumor mass after chemotherapy. Radiotherapy is indicated for rhabdomyosarcomas of the alveolar subtype or in patients with residual tumors after initial treatment [3].

\section{Conclusion}

A rhabdmyosarcoma of the infratemporal fossa may be manifested by nonspecific signs, MRI is the test of choice for assessing the exact extent of the tumor before and after treatment, and CT is better performing in the exploration of destructive bone lesions.

Early identification of the tumor with appropriate therapeutic management according to the age, size and site of the tumor, the histological subtype and the local or remote extension, allows optimization of the healing potential, and decreases the morbidity of therapy.

\section{References}

1. Ferrari A, Dileo P, Casanova M, Bertulli R, Meazza C, Gandola L, et al., Rhabdomyosarcoma in adults. A retrospective analysis of 171 patients treated at a single institution. Cancer. 2003; 98: 571-580.

2. Goto TK, Yoshiura K, Tanaka T, Kanda S, Ozeki S, Ohishi M, et al. A followup of rhabdomyosarcoma of the infratemporal fossa region in adults based on the magnetic resonance imaging findings: case reports. Oral Surg Oral Med Oral Pathol Oral Radiol Endod. 1998; 86: 616-625.

3. Moretti G, Guimarães R, Oliveira KM, Sanjar F, Voegels RL. Rhabdomyosarcoma of the head and neck: 24 cases and literature review. Braz J Otorhinolaryngol. 2010; 76: 533-537.

4. Freling NJ, Merks JH, Saeed P, Balm AJ, Bras J, Pieters BR, et al. Imaging findings in craniofacial childhood rhabdomyosarcoma. Pediatr Radiol. 2010; 40: $1723-1738$.

5. Lee JH, Lee MS, Lee BH, Choe DH, Do YS, Kim KH, et al. Rhabdomyosarcoma of the head and neck in adults: MR and CT findings. AJNR Am J Neuroradiol. 1996; 17: 1923-1928.

6. Allen SD, Moskovic EC, Fisher C, Thomas JM. Adult rhabdomyosarcoma: cross-sectional imaging findings including histopathologic correlation. AJR Am J Roentgenol. 2007; 189: 371-377.
Austin J Radiol - Volume 5 Issue 1 - 2018

ISSN : 2473-0637 | www.austinpublishinggroup.com

Toufga et al. (C) All rights are reserved
Citation: Toufga Z, Fikri M and Jiddane M. Rhabdomyosarcoma of the Infratemporal Fossa: A Case Report. Austin J Radiol. 2018; 5(1): 1080. 